\title{
Anomalies in the antiferromagnetic phase of metamagnets
}

\author{
M. Pleimling and W. Selke \\ Institut für Theoretische Physik B, Technische Hochschule, D-52056 Aachen, Germany
}

\begin{abstract}
Motivated by recent experiments on the metamagnet $\mathrm{FeBr}_{2}$, anomalies of the magnetization and the specific heat in the antiferromagnetic phase of related spin models are studied systematically using Monte Carlo simulations. In particular, the dependence of the anomalous behavior on competing intralayer interactions, the spin value and the Ising-like anisotropy of the Hamiltonian is investigated. Results are compared to experimental findings on $\mathrm{FeBr}_{2}$.
\end{abstract}

$05.50+\mathrm{q}, 75.30 . \mathrm{Kz}, 75.40 . \mathrm{Mg}$

Typeset using REVTEX 


\section{Introduction}

$\mathrm{FeCl}_{2}$ and $\mathrm{FeBr}_{2}$ are much studied metamagnets of Ising type. $\mathrm{H}^{\mathrm{B}}$ The magnetic field $(H)$-temperature $(T)$ phase diagram displays an antiferromagnetically ordered phase, with the transition to the paramagnetic phase being of first order at low temperatures and of second order at higher temperatures and lower fields. In the antiferromagnetic phase the spins of the iron ions are aligned ferromagnetically in the triangular layers perpendicular to the c-axis; along that axis there is an antiparallel ordering of the spins.

In $\mathrm{FeCl}_{2}$, the two kinds of transition meet at a tricritical point. For $\mathrm{FeBr}_{2}$, a possible decomposition of the tricritical point into a critical end point and a bicritical end point has been discussed, in the context of the recent experimental discovery of lines of anomalies in

the antiferromagnetic phase. 110 In particular, the specific heat as well as the temperature derivative of the magnetization and the order parameter may display, at fixed field and varying temperature, shoulders or maxima below the transition to the paramagnetic phase.

The anomalies have been attributed 11 to two crucial ingredients of $\mathrm{FeBr}_{2}$, the effectively weak ferromagnetic intralayer couplings, due to competing antiferromagnetic longer-range interactions, and the highly coordinated interlayer couplings to many equivalent iron ions in adjacent layers, due to the superexchange mediated by the non-magnetic bromide planes. The anomalies have been suggested to reflect the onset of local fluctuations of a second antiferromagnetic phase, the AII phase, which, if becoming eventually thermally stable, would lead to a decomposition of the tricritical point.

In this article, we shall extend the previous analyses to study quantitatively the dependence of the anomalies on different parameters of a realistic model 2 , 13 for $\mathrm{FeBr}_{2}$, namely the competing intralayer couplings, the spin value (being 1 in $\mathrm{FeBr}_{2}$ ), and on the Ising-like anisotropies in the Hamiltonian. Furthermore, the relation between the decomposition of the tricritical point and the anomalies will be discussed, in particular when comparing our Monte Carlo results to recent experimental data and their interpretation.

The layout of the paper is as follows: The Hamiltonian, obtained from spin wave measurements, is introduced and experimental findings are outlined in Sect. II. Then results 
on related Ising models are presented, clarifying the influence of the competing interactions and the spin value, followed by a section on the anisotropic Heisenberg model. In Sect. V, the comparison to experiments is given. Finally, a brief summary concludes the article.

\section{Realistic Hamiltonian for $\mathrm{FeBr}_{2}$}

The compound $\mathrm{FeBr}_{2}$ has the hexagonal structure shown in Fig. 1, with the magnetic iron ions forming triangular layers perpendicular to the $\mathrm{c}^{-}$-axis (corresponding to the $\mathrm{z}^{-}$-axis of Cartesian coordinates). Based on spin-wave analyses 12 , 13 , the low temperature magnetic properties of $\mathrm{FeBr}_{2}$ may be obtained from an effective anisotropic Heisenberg Hamiltonian for the iron ions

$$
\mathcal{H}=\sum_{i>j}\left\{-\frac{1}{\eta} J_{i j} S_{i}^{z} S_{j}^{z}-J_{i j}\left(S_{i}^{x} S_{j}^{x}+S_{i}^{y} S_{j}^{y}\right)\right\}+\sum_{i} D\left\{\left(S_{i}^{z}\right)^{2}-\frac{2}{3}\right\}-H \sum_{i} S_{i}^{z}
$$

with the spin value $S=1$. The first term describes exchange interactions between spins in the same triangular layer and adjacent layers. Two different sets of interactions have been proposed for the intralayer couplings, with ferromagnetic nearest neighbor interactions, $J_{1}$, and competing antiferromagnetic interactions, extending either up to only next-nearest neighbors $12, J_{2}$,

$$
J_{1} / k_{B}=7.3 \mathrm{~K} \text { and } J_{2} / k_{B}=-2.4 \mathrm{~K}
$$

or up to third neighbors in the triangular layers $13, J_{3}$,

$$
J_{1} / k_{B}=4.8 \mathrm{~K}, J_{2} / k_{B}=-0.1 \mathrm{~K} \text { and } J_{3} / k_{B}=-1.0 \mathrm{~K}
$$

The interlayer coupling has been determined unambiguously to be

$$
J_{t}^{\prime} / k_{B}=-2.9 \mathrm{~K}
$$

denoting the total exchange to the adjacent iron layer. Taking into account the ten equivalent superexchange paths, as mediated by the bromide planes, each individual bond between neighboring layers is expected to contribute $J^{\prime} / k_{B}=-0.29 \mathrm{~K}$ (see Fig. 2). 
The Ising-type anisotropy, $\eta=0.7812$, in the first term of the Hamiltonian (1) is enhanced by the second term, describing a single-ion anisotropy with the easy axis of the spin along the $\mathrm{z}$-axis. Here, $D$ is the energy difference between the doublet and singlet in the lowest triplet of an iron ion, with $D / k_{B}=-10.7 \mathrm{~K}$ (for the intralayer couplings of Eq. (2)) or $D / k_{B}=-12 \mathrm{~K}$ (for the intralayer couplings of Eq. (3)).

The third term in Eq. (1) describes the effect of the magnetic field, $H$, applied along the c-axis, i.e. in z-direction.

Fig. 3 shows the $H-T$ phase diagram of $\mathrm{FeBr}_{2}$ determined from measurements of the magnetization期, dynamic susceptibility 5 , and specific heat 6 Varying temperature, at fixed field, all three quantities or their temperature derivatives display in the antiferromagnetic phase unusual behavior in the form of shoulders or maxima at about the same temperature $T_{a}(H)$, locating the anomaly line. That line seems to evolve from the tricritical point. Note that it has been alternately suggested that the anomaly line represents, at sufficiently large magnetic fields, a true phase boundary line between different antiferromagnetic orderings. In that case, one may expect, from mean-field considerations, the anomaly line to emerge from the (bi)critical point at the end of the additional phase boundary lind11 point having turned into a critical end point.

In Fig. $3, T_{p}$ denotes a line in the paramagnetic phase at which the dynamic susceptibility and the specific heat $\mathrm{l}$ show a maximum, when changing temperature at fixed field. It may seem to be conceivable that this line also evolves from the tricritical point (or critical end point), but this aspect has not been investigated experimentally in detail.

In the following, we shall study simplified models based on the anisotropic Heisenberg Hamiltonian for $\mathrm{FeBr}_{2}$, Eq. (1), to clarify which of its features may enhance (or weaken) the anomalies and, possibly, decompose the tricritical point. So far, previous recent analyse 91 . 1 dealt with Ising variants of (1), where $S=1 / 2$. Perhaps most importantly, the crucial importance of the high interlayer coordination, driving the system close to a mean-field type behavior and thereby inducing local thermal excitations of AII-type for weak intralayer exchange couplings, was established. 1.1 Taking merely interactions to the 
geometric nearest neigbor spins in adjacent layers, no anomalies were found 9 . 1 (in that case, fluctuations also destroy the AII phase, and hence the tricritical point does not decompose, as had been seen in simulations 15 ). Here, we shall elaborate systematically on the role of the other parameters in the Hamiltonian, specifically on that of the competing intralayer exchange couplings, the spin value, and the spin anisotropy. Thereby, we shall approach a rather realistic description of $\mathrm{FeBr}_{2}$. A full analysis of the complete model, Eq. (1), is, however, beyond the scope of our study. In addition, such an analysis may provide only an integral and thence a rather limited insight into the relevant ingredients leading to anomalies in the antiferromagnetic phase of metamagnets.

\section{Ising models}

We shall first approximate the Hamiltonian (1) by Ising models, to elucidate quantitatively the importance of the competing intralayer couplings as well as the spin value in stabilizing the anomalies in the magnetization and the specific heat.

\section{A. Spin $1 / 2$}

Let us consider the $S=1 / 2-$ Ising Hamiltonian

$$
\mathcal{H}=-J^{\prime} \sum_{\langle N N\rangle} S_{i} S_{j}-J_{1} \sum_{N N} S_{i} S_{j}-J_{2} \sum_{N N N} S_{i} S_{j}-J_{3} \sum_{3 N N} S_{i} S_{j}-H \sum_{i} S_{i}
$$

where $S_{i}$ is an Ising spin on site $i$, with spin value $1 / 2$. The exchange interactions $J_{i, j}$ describe, as before, intralayer (extending up to third neighbors in the triangular planes, $J_{1}$, $J_{2}$, and $J_{3}$ ) and interlayer (to the ten equivalent sites in the adjacent plane, $J^{\prime}$ ) couplings. The couplings are normalized by setting $\left|J^{\prime}\right|=1$. To study the effect of the competing interactions in the planes, we usually fix the nearest neighbor interaction $J_{1}$, and vary the two remaining antiferromagnetic couplings, $J_{2}$ and $J_{3}$. According to the two different types

of exchange constants determined experimentally 22,13 , two cases are of special interest: (a) $J_{2}=0, J_{3}<0$, and (b) $J_{2}<0, J_{3}=0$, respectively. To quantify the efficiency of the antiferromagnetic couplings in weakening the effective ferromagnetic nearest neighbor interactions, 
we also investigated the case (c) $J_{2}=0, J_{3}=0$, changing $J_{1}$.

We simulate systems with $K$ layers, each one consisting of $L \times L$ spins, using full periodic boundary conditions. Typically, we choose $K=L=20$ (to check finite size effects, $K$ and $L$ ranged from 10 to 40 ). For equilibration, $10^{4}$ Monte Carlo steps per site (MCS) were used; averages were taken over the following $2 \times 10^{4} \mathrm{MCS}$. To improve the statistics and to calculate error bars, we performed simulations for ten realizations, with different random numbers, at a given field, $H$, and temperature, $T /\left|J^{\prime}\right|$. We computed several quantities of interest, in particular the energy, $E$, the specific heat, $C$ (both from energy fluctuations and by differentiating the energy with respect to the temperature), the magnetization per layer, $M(i)$, and related quantities such as the total magnetization, $M$, the sublattice magnetizations, $M_{1}$ and $M_{2}$, referring to the odd and even layers, and the order parameter $M_{s}=\left(M_{1}-M_{2}\right) / 2$. To take into account phase shifts or flips of entire spin layers, we usually computed the absolute values of the total magnetization and the order parameter (which will be denoted by $M_{s}$ in the following). In a few cases, we also determined correlation lengths from standard spin-spin correlation functions.

In case (a), the ground state, at $T=0$ and $H<H_{c 0}=20\left|J^{\prime}\right|$, is the antiferromagnetic structure, $M_{1}=1$ and $M_{2}=-1$, assuming $\left|J_{3}\right|<\frac{1}{2} J_{1}$ (otherwise, more complicated spin configurations are stabilized 16 , due to the competing interactions along the axes of the triangular layers). Results of the simulations for that case, fixing the field at $H=0.9 H_{c 0}$ and changing the temperature, are depicted in Fig. 4, showing the specific heat, the order parameter, and the temperature derivative of the total magnetization for various values of $J_{3}$. In accordance with the experimental findings 13 for $\mathrm{FeBr}_{2}, J_{1}$ has been set equal to $16.75\left|J^{\prime}\right|$ (recall that the values obtained from the spin wave analysis are $J_{1} / k_{B}=4.8 / \eta=6.2 \mathrm{~K}$ and $\left.J^{\prime} / k_{B}=-0.29 / \eta=-0.37 \mathrm{~K}\right)$.

In the finite Monte Carlo system, the transition to the paramagnetic phase, at $T_{N}$, man- 
ifests itself, for instance, by a maximum in the specific heat and a drastic decrease in the order parameter $M_{s}$, leading to singularities in the thermodynamic limit. More interestingly, anomalous behavior is seen in Fig. 4 to occur well below that transition. For example, the specific heat and the temperature derivative of the magnetization display shoulders or maxima, becoming more pronounced with increasing antiferromagmetic interactions $J_{3}$. The anomalies vanish for smaller values of $J_{3}$ (not shown in Fig. 4).

Let us briefly recall the physical picture underlying the anomalies, as has been obtained from mean-field theory of Ising metamagnets with only nearest neighbor ferromagnetic intralayer couplings.11 If those couplings are sufficiently weak, compared to the interlayer interactions, a second antiferromagnetic phase, AII, may be formed in between the usual antiferromagnetic phase (AI, with $M_{1}>0$ and $\left.M_{2}<0\right)$ and the paramagnetic phase $\left(M_{1}=M_{2}\right)$, in which both sublattice magnetizations are positive, but different. The AII phase may be thought of balancing the conflicting tendencies of the external field and the antiferromagnetic interlayer couplings, by maintaining, rather small, clusters of 'minus' spins in the even layers. Strong ferromagnetic intralayer interactions tend to disfavor those clusters, thereby suppressing the AII phase. The transition between the AI and AII phases is of first order, with the boundary line evolving from the critical end point on the border line to the paramagnetic phase, and terminating at a (bi)critical point. From that (bi)critical point, a line of anomalies emerges. However, such a line may persist even when there is a tricritical point, provided the ferromagnetic intralayer couplings are still sufficiently small.

Including now competing antiferromagnetic intralayer couplings, one may try to cast them, together with $J_{1}$, in an effective nearest neighbor ferromagnetic interaction, $J_{\text {eff }}$. To elucidate the effect of $J_{3}$ on reducing $J_{e f f}$, we compared our simulational data, case (a), to those for models with only nearest-neighbor ferromagnetic intraplane couplings, case (c), varying $J_{1}$, with $\left|J^{\prime}\right|=1$. In particular, we determined the change in $J_{1}, \delta J_{1}$, needed to reproduce the Néel temperature $T_{N}$, at $H=0$, when $J_{3} \neq 0$. A naive argument of mean- 
field type suggests that $\delta J_{1}=J_{3}$, i.e. $J_{\text {eff }}=J_{1}+J_{3}$. In reality, the antiferromagnetic coupling is much more efficient in lowering the effective interaction (as may be already seen from the analysis of the ground states). For instance, at $J_{1}=16.75\left|J^{\prime}\right|$ and $J_{3}=4.9 J^{\prime}$, we find $\delta J_{1} \approx-8.15\left|J^{\prime}\right|$. Note that an even much stronger reduction in $J_{1}$ is required in reproducing, instead of $T_{N}$, the kind of anomalies present when the antiferromagnetic intraplane couplings are included, see Fig. 5. We find, at $H=0.9 H_{c 0}$, that the value of $J_{3}=4.9 J^{\prime}$ then corresponds to weakening $J_{1}$ from $16.75\left|J^{\prime}\right|$ to roughly $1.5\left|J^{\prime}\right|$. The high efficiency of $J_{3}$ (or $J_{2}$, see below) in lowering the effective ferromagnetic coupling $J_{\text {eff }}$ and hence the ferromagnetic ordering in the layers is crucial, together with the large interlayer coordination, in explaining the experimentally found anomalies in $\mathrm{FeBr}_{2}$.

Note that the anomalies shown in Fig. 4 do not correspond to sharp phase transitions. For instance, they do not seem to give rise to singularities, as one increases the size of the Monte Carlo systems (going from $L=K=10$ to 40, e.g., the height of the 'anomalous' maximum in $\mathrm{C}$ below $T_{N}$, at $H=0.95 H_{c 0}$, does not change significantly, in contrast to the behavior of $C$ close to $T_{N}$, where the peak becomes clearly visible at $L=K=20$, increasing furthermore for the larger systems), nor is there any indication of hysteresis (by crossing the anomalies from different directions in the field-temperature phase plane). Indeed, the anomalies may be interpreted as reflecting the onset of local ordering of AII-type, with the long-range order of the AII-phase being, possibly always, destroyed by fluctuations. They may be also illustrated by monitoring typical equilibrium Monte Carlo configurations.

Fixing $J_{3}$ and varying the external field, one may map the anomaly line, $T_{a}(H)$. Examples for a specific value of $J_{3}, J_{3} / J_{1}=-0.29$, chosen to be close, but, in order to identify easily the location of the anomalies, somewhat larger than that obtained for $\mathrm{FeBr}_{2}$, are depicted in Fig. 6. Obviously, the anomalies become stronger upon increasing the field $H$. However, they seem to go over into singularities only at the tricritical point on the phase boundary to the paramagnetic phase, as concluded from analyses of the types mentioned 
above, for fields in the range in between $0.8 H_{c 0}$ and $0.95 H_{c 0}$ (strictly speaking, if there is a decomposition of the tricritical point, then the critical end point on the transition line to the paramagnetic phase and the critical point at the end of the phase boundary between the AI and AII phases would be very close to each other). The Monte Carlo data for locating the anomaly line are summarized in Fig. 7, depicting the phase diagram in the $H-T$ plane, at $J_{3} / J_{1}=-0.29$. $T_{a}$ has been determined from the anomaly in the specific heat, in good agreement with the corresponding estimates obtained from the magnetizations.

We also identified the line, $T_{p}$, in the paramagnetic phase, at which the specific heat $C$, at fixed fields, displays a maximum as a function of temperature, see Fig. 7. The maximum is believed to reflect a disordering in the triangular layers. 17 In close agreement with the experimental findings on $\mathrm{FeBr}_{2}$, see Fig. 3, the line seems to evolve from the tricritical point. This feature may be, however, accidental. In mean-field theory, $T_{p}$ intersects the boundary of the antiferromagnetic phase, $T_{N}$, at some point, which is, in general, not related to the tricritical or critical end point. In the simulations, the height of the maximum in $C$ does not change drastically on approach to the boundary of the antiferromagnetic phase, indicating a non-critical behavior, see Fig. 8. It may be wortwhile to clarify this aspect by determining the location of $T_{p}$ for different values of $J_{3}$, where it may be easier to disentangle the intersection point, of $T_{p}$ and $T_{N}$, and the tricritical point. Note that the specific heat $C$, fixing the temperature and varying the field, exhibits in the paramagnetic phase a maximum at about $T_{p}$ as well, see Fig. 7 , in accordance with recent experimental findings.18

In case (b), i.e. $J_{2}<0, J_{3}=0$, similar conclusions hold. To describe experimental data on $\mathrm{FeBr}_{2}$, we may choose $J_{1}=25.1\left|J^{\prime}\right|$ and $J_{2}=8.4 J^{\prime} 12$ The antiferromagnetic couplings lead to a weakening of an effective ferromagnetic intralayer interaction, giving eventually rise to AII-type excitations in the even or 'minus' layers which cause the anomalies in the specific heat $C$ and magnetizations. Actually, $J_{3}$ is slightly more efficient than $J_{2}$ in reducing $J_{\text {eff }}$, as seen when adjusting $J_{2}$, with $J_{1}=25.1\left|J^{\prime}\right|$, to reproduce 
$T_{N}\left(H=0, J_{1}=16.75\left|J^{\prime}\right|, J_{3}=4.9 J^{\prime}\right)$. The Néel temperature is realized, when $J_{2}=10.3 J^{\prime}$ (being not far from the experimentally determined value). The anomalies for the two sets of parameters do not differ much, see Fig. 5 , demonstrating that both types of couplings, $J_{2}$ as well as $J_{3}$, have a comparable effect on the anomalies, although they are of quite distinct physical character (frustration on triangles, $J_{2}$, or competition along the axes of the triangular planes, $\left.J_{3}\right)$. It should be emphasized that, in general, frustration or competition is not really needed for obtaining the anomalies in the antiferromagnetic phase of metamagnets: $J_{\text {eff }}$ has to be sufficiently weak.

In addition, we determined in which way the ratio of the tricritical temperature $T_{t}$ to the Néel temperature $T_{N}(H=0)$ depends on the strength of the antiferromagnetic intraplane interactions, for the cases (a) and (b), fixing $J_{1}$ at the value appropriate for $\mathrm{FeBr}_{2}$. The ratio decreases with increasing $J_{2}$ or $J_{3}$ (i.e. decreasing $J_{\text {eff }}$, see also results from mean-field theory, simulations and high-temperature series expansions 3.11 -1920). For instance, in case (a), the ratio varies in between about 0.6 and 0.4 , when changing $J_{3}$ from $3.3 \mathrm{~J}^{\prime}$ to $6.5 \mathrm{~J}^{\prime}$, with $J_{1}=16.75\left|J^{\prime}\right|$. Similarly, the ratio may be lowered to about 0.32 , when increasing $J_{2}$ to $12.1 J^{\prime}$, with $J_{1}=25.1\left|J^{\prime}\right|$.

\section{B. Spin 1}

We now consider the $S=1$ Ising Hamiltonian, see Eq. (4), where each spin can take the values 0,1 or -1 . Compared to the situation with $S=1 / 2$, thermal fluctuations are facilitated, reducing the transition temperatures and resulting in more pronounced anomalies.

In particular, we studied the case $J_{1}=16.75\left|J^{\prime}\right|$ and $J_{3}=4.9 J^{\prime}$, with $J_{2}=D=0$, setting $\left|J^{\prime}\right|=1$, as before for $S=1 / 2$. Results are displayed in Fig. 9, showing the specific heat $C$ versus temperature at various fields, compare to Fig. 6. Clearly, at larger fields a maximum shows up below $T_{N}$; that anomalous behavior in $C$ is corraborated by similar properties of the magnetizations, for instance, of $d M_{s} / d T$. 
The intersection point of the line of anomalies and the boundary to the paramagnetic phase is supposedly the tricritical point (again, we found no evidence for a transition of first order between the $\mathrm{AI}$ and AII phases). The ratio $T_{t} / T_{N}(H=0)$ is roughly 0.5 , as is the case for $S=1 / 2$ with the same values of $J_{1}$ and $J_{3}$. Note that the Néel temperature, $T_{N}(H=0)$, is, however, compared to its value for the $S=1 / 2$ Ising model, lower by nearly 30 percents. Expressing the coupling constants in terms of Kelvin, one easily sees that one moves in the case of $S=1$ much closer towards the experimentally determined Néel temperature in $\mathrm{FeBr}_{2}$, see below.

\section{Anisotropic Heisenberg models}

We now proceed to the anisotropic $S=1$ Heisenberg model, given in Eq. (1). In a semiclassical descriptione 2 of such a model, the $\mathrm{z}^{-}$-component of the spin, of lenght 1 , is discretized, taking the values $S^{z}=0,1$ or -1 . If $S^{z}=0$, then the spin can rotate, like a classical vector, in the xy-plane, see Fig. 10.

As in the Ising case, we studied especially the case $J_{1}=16.75\left|J^{\prime}\right|, J_{2}=0$, and $J_{3}=4.9 J^{\prime}$. Putting $D=0$ and $\eta=0.78$, the simulational data for the specific heat and the magnetizations are very close to those for the corresponding $S=1$ Ising model. For example, at $H=0.9 H_{c 0}$, the critical and anomaly temperatures are, in the Heisenberg model, lower by roughly one percent. The specific heat is essentially identical to that shown in Fig. 9 for the Ising model. In turn, the derivative of the order parameter, $d M_{s} / d T$, for the Heisenberg model, see Fig. 11, agrees very well with that for the Ising case.

By turning on the single ion anisotropy, $D$, the critical temperature is shifted towards higher values, and the anomalies are somewhat suppressed. In effect, by discriminating $S^{z}=0$, one approaches the $S=1 / 2$ Ising model. 
In general, the thermal properties of semiclassical $S=1$ Heisenberg models seem to resemble quite closely those of the corresponding $S=1$ Ising models. Deviations are due to spins with vanishing $\mathrm{z}$-component, which may provide, e.g., additional energy contributions. Obviously, the different spin components are not decoupled, leading, perhaps, to intriguing effects. However, it is beyond the scope of our study, to explore this class of models extensively (in passing, we may mention our simulational results on the $S=1$ Heisenberg model with ferromagnetic couplings between neighboring spins on a square lattice. They indicate non-critical energy contributions stemming from the xy-components of the spins, leading to a minor lowering in the transition temperature, compared to that of the corresponding Ising model).

Note that the discretization of the $\mathrm{z}$-component of the spin is crucial in reproducing the anomalous behavior found in $\mathrm{FeBr}_{2}$. A classical Heisenberg model with spins of fixed length, but arbitrary orientation, is not expected to show any tendency towards forming, even locally, the AII phase. Indeed, preliminary simulations on such Heisenberg models did not show anomalies in the specific heat or the magnetizations.

\section{Comparison with experiments}

A typical phase diagram of a simplified, but supposedly rather realistic model for $\mathrm{FeBr}_{2}$ is depicted in Fig. 7. Obviously, it resembles quite closely the experimental phase diagram, see Fig. 3. However, for a quantitative comparison, a few points need to be viewed with care.

Experimentally2 2 国, the Néel temperature $T_{N}(H=0)$ is found to be $14.2 \mathrm{~K}$. Using the two sets of coupling parameters as obtained from spin wave analyses, see Eqs. (2) and (3), $T_{N}(H=0)$ moves towards that temperature from above, by going from the Ising models with $S=1 / 2$ to those with $S=1$ and finally to the anisotropic Heisenberg models (both sets give only slightly different transition temperatures). Indeed, the $S=1 / 2$ Ising models, for 
both sets of parameters, overestimate $T_{N}(H=0)$ by almost a factor of 2 (note that previous analyses for $\mathrm{FeBr}_{2}$ were restricted to that case). For the semiclassical $\mathrm{S}=1$ Heisenberg model, $T_{N}(H=0)$ is about $20 \mathrm{~K}$, i.e. it is still too high. That remaining difference may be partly due to a temperature dependence in the effective strength of the single-ion anisotropy, $D$, as had been observed in $\mathrm{FeCl}_{2} \mathbb{1}$, with $D$ becoming smaller at higher temperatures, thereby tending to lower the Néel temperature (in $\mathrm{FeBr}_{2}, D$ had been estimated only at a single, low temperature).- Similarly, the ratio of $T_{t} / T_{N}$ is not reproduced quantitatively by the model description. While it is about 0.34 in $\mathrm{FeBr}_{2}$, the simulations yield such low values, e.g., when increasing the antiferromagnetic intraplane interactions beyond the experimentally determined values, as discussed above.

Of course, the deviations from the experimental results might be due to simplifications in the model and its treatment, such as neglect of dipolar interactions between the spins (their relevance may be seen from the broad two-phase region at low temperatures; they also would affect the problem of distinguishing the external, used in experiments, from the internal magnetic field, used in the simulations) and neglect of much of the quantum nature of the spins.

As stated before, the main aim of our study is to discuss the origin and character of the anomalies in the antiferromagnetic phase. While the model description gives no evidence for a sharp transition from the AI to the AII phase, such a possibility has been suggested recently based on measurements of the specific heat 6 and, using neutron scattering techniques, the order parameter $M_{s}$ 月. In particular, the specific heat, as a function of temperature, showed a sharp peak superposed on the broad shoulder or maximum well below the transition to the paramagnetic phase 6 , becoming sharper with increasing field. However, these findings have been questioned later 18 . Indeed, no peaks were detected, but only the shoulders or maxima, in agreement with the model calculations. 
In addition, the experimental data for $M_{s} 7$ were interpreted in favour of a real transition between the AI and AII phases. The data, at different fields, were plotted against $T / T_{N}(H)$

and against $T / T_{a}(H)$. I In the former case, data separation was observed for $T<0.95 T_{N}$, while in the latter case, the data seemed to fall on one 'universal' curve for $T<T_{a}$.

In Fig. 12, we show the corresponding plots of the Monte Carlo data for the $S=1 / 2$ Ising model with $J_{1}=16.75\left|J^{\prime}\right|, J_{2}=0$ and $J_{3}=4.9 J^{\prime}$. Indeed, the behavior is quite similar to that found in the experiments, with a clear separation of the order parameter for different fields in the predicted ranges of temperatures. Because our analyses, see above, give no indication for a sharp phase transition at $T_{a}$, at least for the fields shown in Fig. 12, we, however, tend to conclude that this type of data presentation is not suitable for providing convincing evidence for the suggested phase transition.

\section{Summary}

Motivated by recent experiments on the metamagnet $\mathrm{FeBr}_{2}$, anomalies in the antiferromagnetic phase of Ising-type models, closely related to the realistic Hamiltonian for that magnet as determined from spin wave analyses, have been studied using Monte Carlo techniques.

We clarified which ingredients of the Hamiltonian are relevant for the anomalous properties, such as broad shoulders or maxima in the specific heat and magnetizations well below the transition to the paramagnetic phase. In general, the anomalies can be attributed to local thermal excitations of the AII phase, due to high coordination of spins in adjacent layers and weak effective ferromagnetic intraplane couplings. We demonstrated quantitatively, that, in an Ising description, the anomalies are enhanced by competing antiferromagnetic intralayer interactions, extending up to third neighbors, and by the spin value, $S=1$ in the case of $\mathrm{FeBr}_{2}$. Going from the Ising model to an anisotropic $S=1$ semiclassical Heisenberg model leads to only minor changes in the specific heat and the magnetizations parallel to 
the direction of the applied field. The discretization of the $\mathrm{z}-$-component of the spin plays a major role in obtaining the anomalies.

Finally, we compared results of our simulations to experimental findings. The simulational data suggest that the anomalies usually do not correspond to a sharp phase transition. Conflicting interpretations of experiments may be viewed with much care.

\section{Acknowledgements}

It is a pleasure to thank W. Kleemann, Ch. Binek and O. Petracic for very useful discussions on their stimulating experiments on $\mathrm{FeBr}_{2}$. We should like to thank H. Aruga Katori for sending us intriguing experimental results prior to publication, and M. Mönnigmann for calculations supplementary to the previous analysis of the mean-field theory. 


\section{REFERENCES}

${ }^{1}$ R. J. Birgeneau, W. B. Yelon, E. Cohen, and J. Makovsky, Phys. Rev. B 5, 2607 (1972).

${ }^{2}$ A. R. Fert, P. Carrara, M. C. Lanusse, G. Mischler, and J. P. Redoules, J. Phys. Chem. Solids 34, 223 (1973).

${ }^{3}$ J. M. Kincaid and E. G. D. Cohen, Phys. Rep. 22C, 57 (1975).

${ }^{4}$ E. Stryjewski and N. Giordano, Adv. Phys. 26, 487 (1977)

${ }^{5}$ M. M. P. de Azevedo, Ch. Binek, J. Kushauer, W. Kleemann, and D. Bertrand, J. Magn. Magn. Mater. 140-144, 1557 (1995).

${ }^{6}$ H. Aruga Katori, K. Katsumata, and M. Katori, Phys. Rev. B 54, R9620 (1996).

${ }^{7}$ K. Katsumata, H. Aruga Katori, S. M. Shapiro, and G. Shirane, Preprint (1996).

${ }^{8}$ O. Petracic, Ch. Binek, and W. Kleemann, J. Appl. Phys. to be published (1997).

${ }^{9}$ W. Selke and S. Dasgupta, J. Magn. Magn. Mater. 147, L245 (1995).

${ }^{10}$ K. Held, M. Ulmke, and D. Vollhardt, Mod. Phys. Lett B 10, 203 (1996).

${ }^{11}$ W. Selke, Z. Phys. B 101, 145 (1996).

${ }^{12}$ W. B. Yelon and C. Vettier, J. Phys. C8, 2760 (1975).

${ }^{13}$ S. Pouget, Thèse, I.N.S.A. Toulouse, France (1993).

${ }^{14}$ L. Hernández, H. T. Diep, and D. Bertrand, Europhys. Lett. 21, 711 (1993).

${ }^{15}$ H. J. Herrmann and D. P. Landau, Phys. Rev. B 48, 239 (1993).

${ }^{16}$ Y. Tanaka and N. Uryû, Progr. Theor. Phys. 55, 1356 (1976).

${ }^{17}$ L. Hernández, H. T. Diep, and D. Bertrand, Phys. Rev. B 47, 2602 (1993).

${ }^{18}$ A. Biederstedt, Ch. Binek, and W. Kleemann, in preparation 
${ }^{19}$ D. P. Landau, Chin. J. Phys. 15, 116 (1977).

${ }^{20}$ F. Harbus and H. E. Stanley, Phys. Rev. B 8, 1141 (1973).

${ }^{21}$ I. Vilfan and S. Galam, Phys. Rev. B 34, 6428 (1986). 


\section{FIGURES}

FIG. 1. Sketch of the crystal structure of $\mathrm{FeBr}_{2}$, showing the $\mathrm{Fe}^{2+}$ (full circles) and $\mathrm{Br}^{-}$(open circles) ions.

FIG. 2. The triangular iron planes, with the ten equivalent neighbors (full symbols) in the adjacent layer below.

FIG. 3. Approximate experimental phase diagram, based on measurements of the specific heat and magnetizations, see Refs. 5 and $6 . T_{a}$ denotes the anomaly line in the antiferromagnetic phase, $T_{p}$ indicates the location of maxima in the specific heat, at fixed magnetic fields, in the paramagnetic phase, and $T_{N}$ the boundary to the paramagnetic phase. At low temperatures, the transition of first order leads to a 2-phase region.

FIG. 4. Monte Carlo data of (a) the specific heat $C$, (b) the order parameter $M_{s}$, and (c) the temperature derivative of the total magnetization $d|M| / d T$ versus $T /\left|J^{\prime}\right|$ for the $S=1 / 2$ Ising model with $J_{1}=16.75\left|J^{\prime}\right|$ and $H=0.9 H_{c 0}$ at various values of $J_{3}$. Systems with $K=L=20$ spins are considered. Here and in the following figures, error bars are only shown when they are larger than the sizes of the symbols.

FIG. 5. Simulational data of the specific heat $C$ versus temperature $T /\left|J^{\prime}\right|$, at $H=0.9 H_{c 0}$, with (a) $J_{1}=16.75\left|J^{\prime}\right|, J_{3}=4.9 J^{\prime}$, (b) $J_{1}=25.1\left|J^{\prime}\right|, J_{2}=10.3 J^{\prime}$, and (c) $J_{1}=J_{\text {eff }}=8.6\left|J^{\prime}\right|$, where the Néel temperature $T_{N}(H=0)$ is approximately the same in all three cases. Systems with $K=L=20$ spins are considered.

FIG. 6. The specific heat $C$ as a function of temperature $T /\left|J^{\prime}\right|$ for the $S=1 / 2$ Ising model with $J_{1}=16.75\left|J^{\prime}\right|$ and $J_{3}=4.9\left|J^{\prime}\right|$ at various fields. Systems with $K=L=20$ spins are simulated. 
FIG. 7. Phase diagram in the field $(H)$-temperature $(T)$ plane of the $S=1 / 2$ Ising model with $J_{1}=16.75\left|J^{\prime}\right|$ and $J_{3}=4.9 J^{\prime}$. The anomalies, $T_{a}$, in the antiferromagnetic phase, determined from the specific heat and the magnetizations, are denoted by full circles. The maxima in the specific heat,$T_{p}$, at fixed fields (open) or fixed temperatures (full), in the paramagnetic phase are shown by triangles. Monte Carlo systems with $20 \times 20 \times 20$ spins are simulated.

FIG. 8. Specific heat $C$ versus temperature, $T /\left|J^{\prime}\right|$, at various fields in the paramagnetic phase, see Fig. 7.

FIG. 9. Specific heat $C$ versus temperature $T /\left|J^{\prime}\right|$ for the $S=1$ Ising model with $K=L=20$ spins, at $J_{1}=16.75\left|J^{\prime}\right|$ and $J_{3}=4.9 J^{\prime}$, and various fields.

FIG. 10. Orientations of the spin used in the semiclassical $S=1$ Heisenberg model, with discretization of the $\mathrm{z}$-component and continuous symmetry in the $\mathrm{xy}$-plane.

FIG. 11. Temperature derivative of the order parameter, $d M_{s} / d T$, versus temperature $T /\left|J^{\prime}\right|$ for the anisotropic $S=1$ Heisenberg model with $20 \times 20 \times 20$ spins, for $J_{1}=16.75\left|J^{\prime}\right|, J_{3}=4.9 J^{\prime}$, $D=0$, and $\eta=0.78$, at $H=0.9 H_{c 0}$.

FIG. 12. Order parameter, $M_{s}$, versus reduced temperatures, (a) $T / T_{N}(H)$, and (b) $T / T_{a}(H)$, for the $S=1 / 2$ Ising model with $J_{1}=16.75\left|J^{\prime}\right|, J_{3}=4.9 J^{\prime}$, at various fields, showing ranges of temperatures (see text) where data (almost) collapse or are widely separated, as in experiments on $\mathrm{FeBr}_{2}$, see Ref. 7. Systems with $K=L=20$ spins are simulated. 


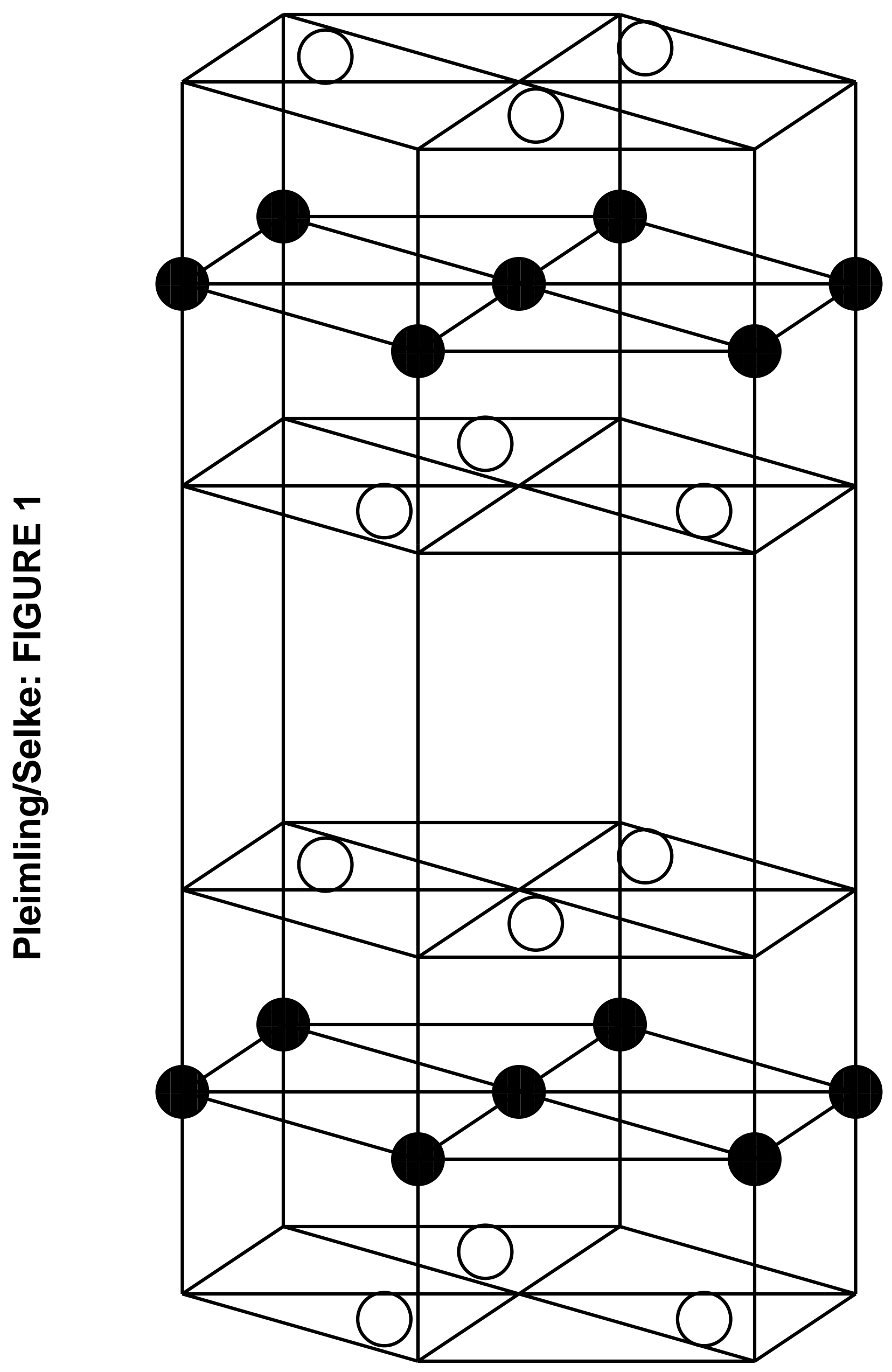




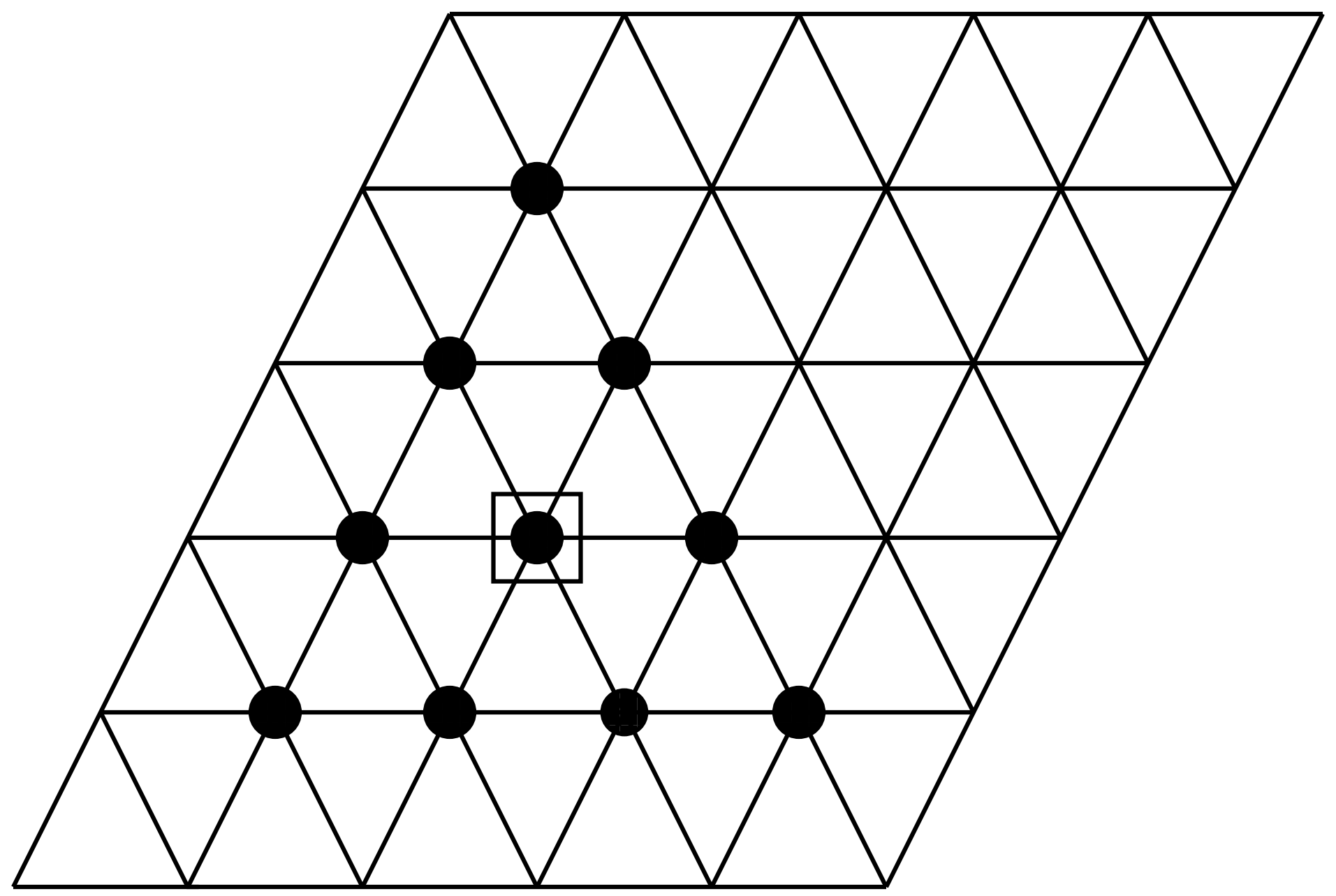

Pleimling/Selke: FIGURE 2 
Pleimling/Selke: FIGURE 3

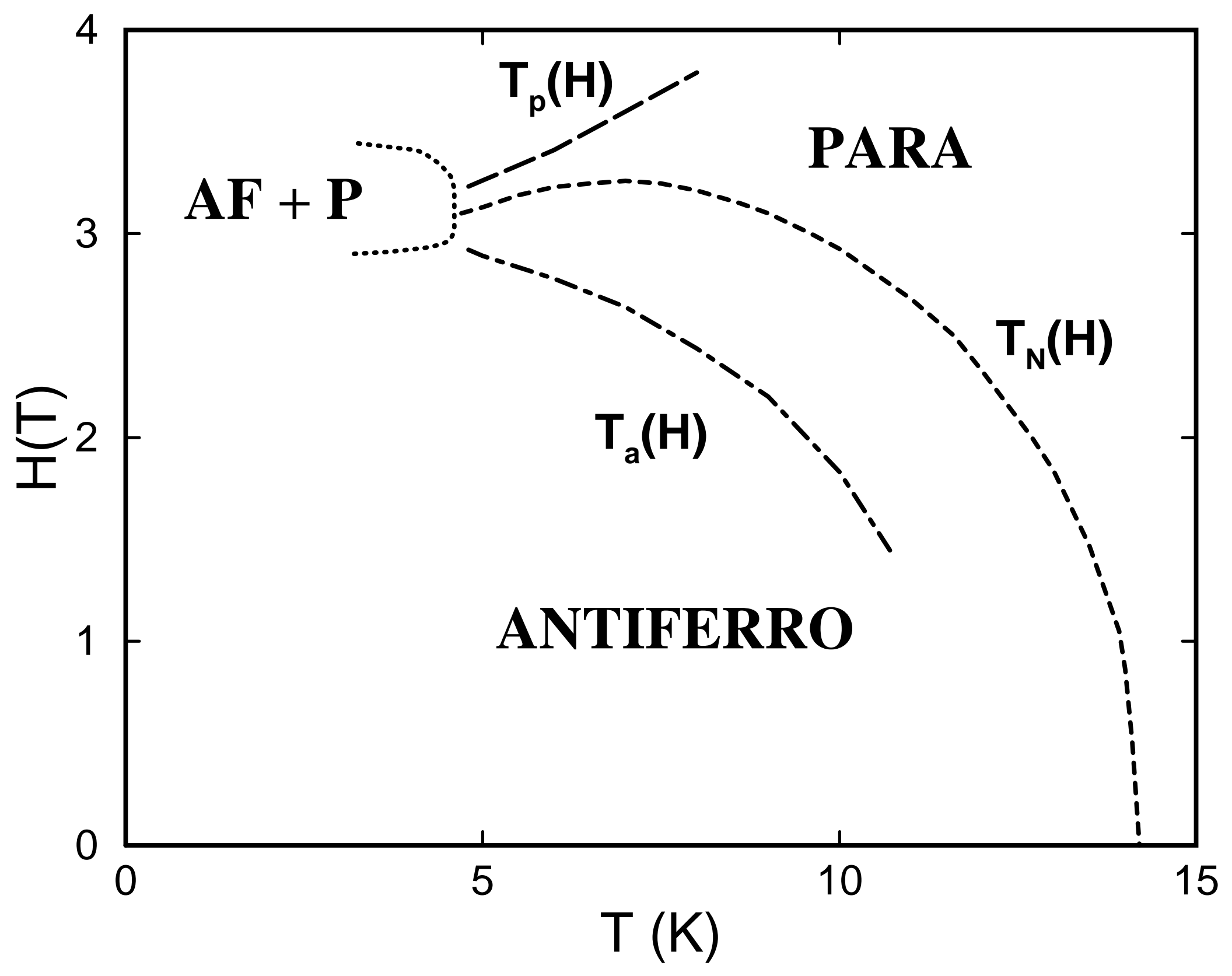


Pleimling/Selke: FIGURE 4a

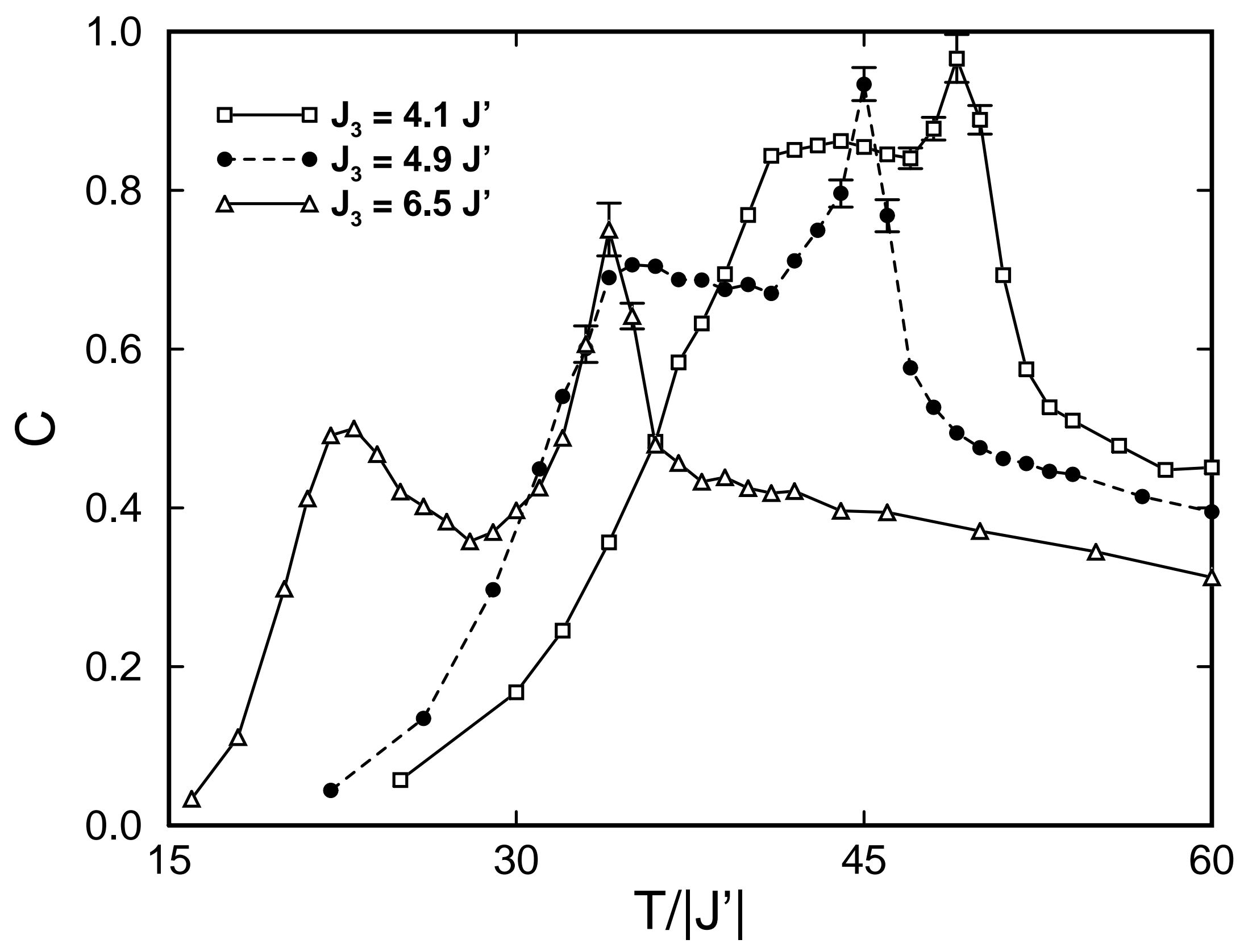


Pleimling/Selke: FIGURE 4b

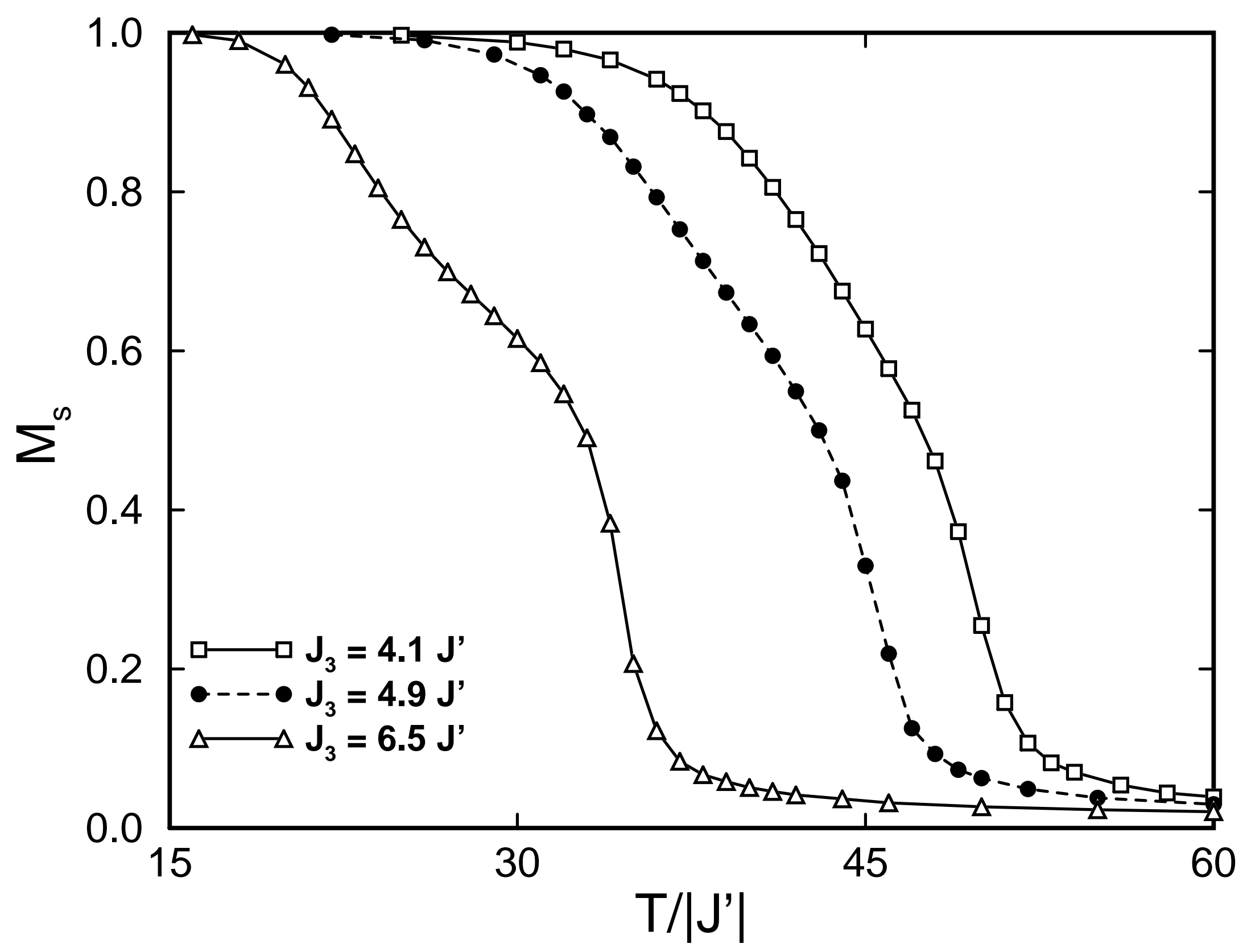


Pleimling/Selke: FIGURE 4c

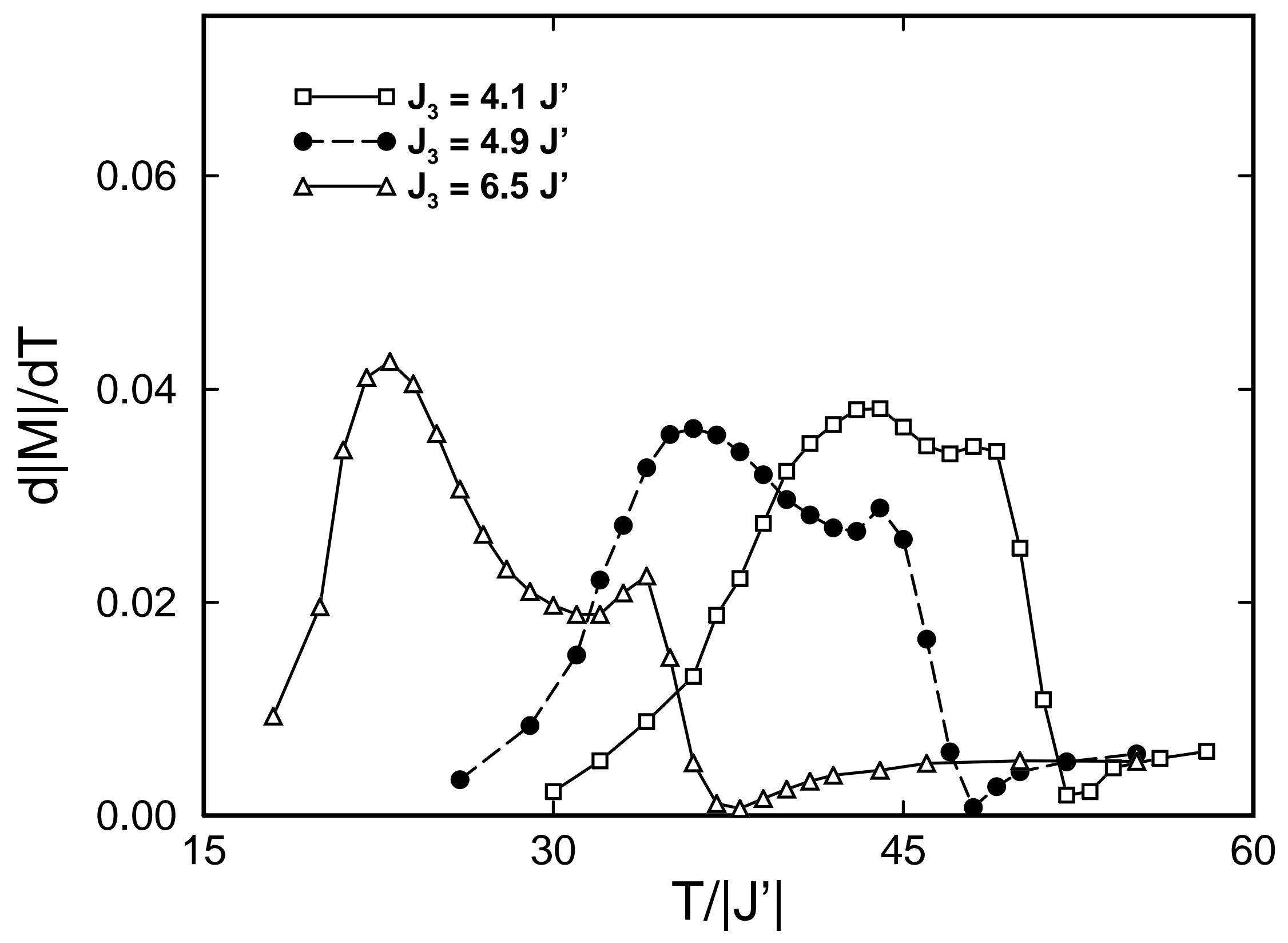




\section{Pleimling/Selke: FIGURE 5}

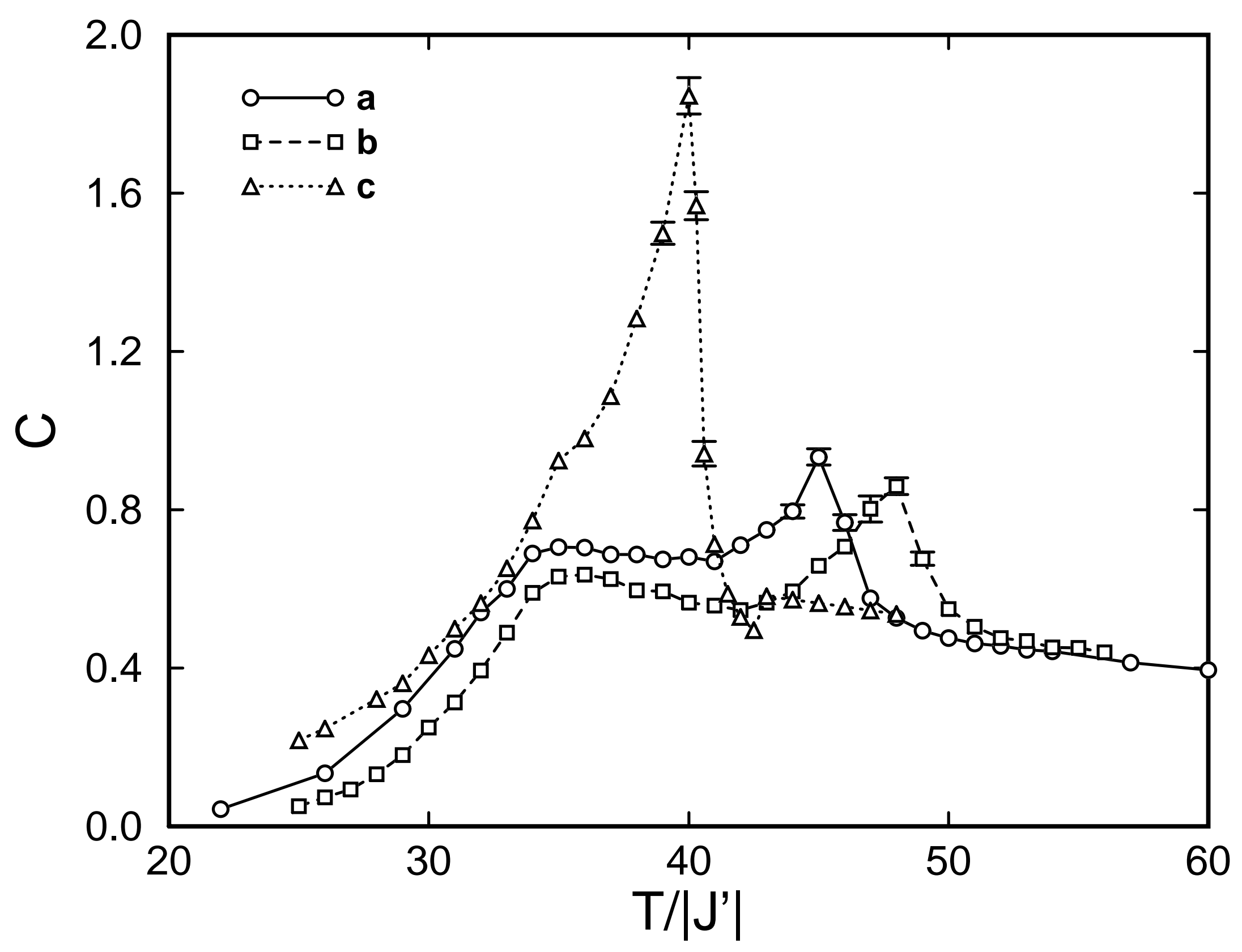




\section{Pleimling/Selke: FIGURE 6}

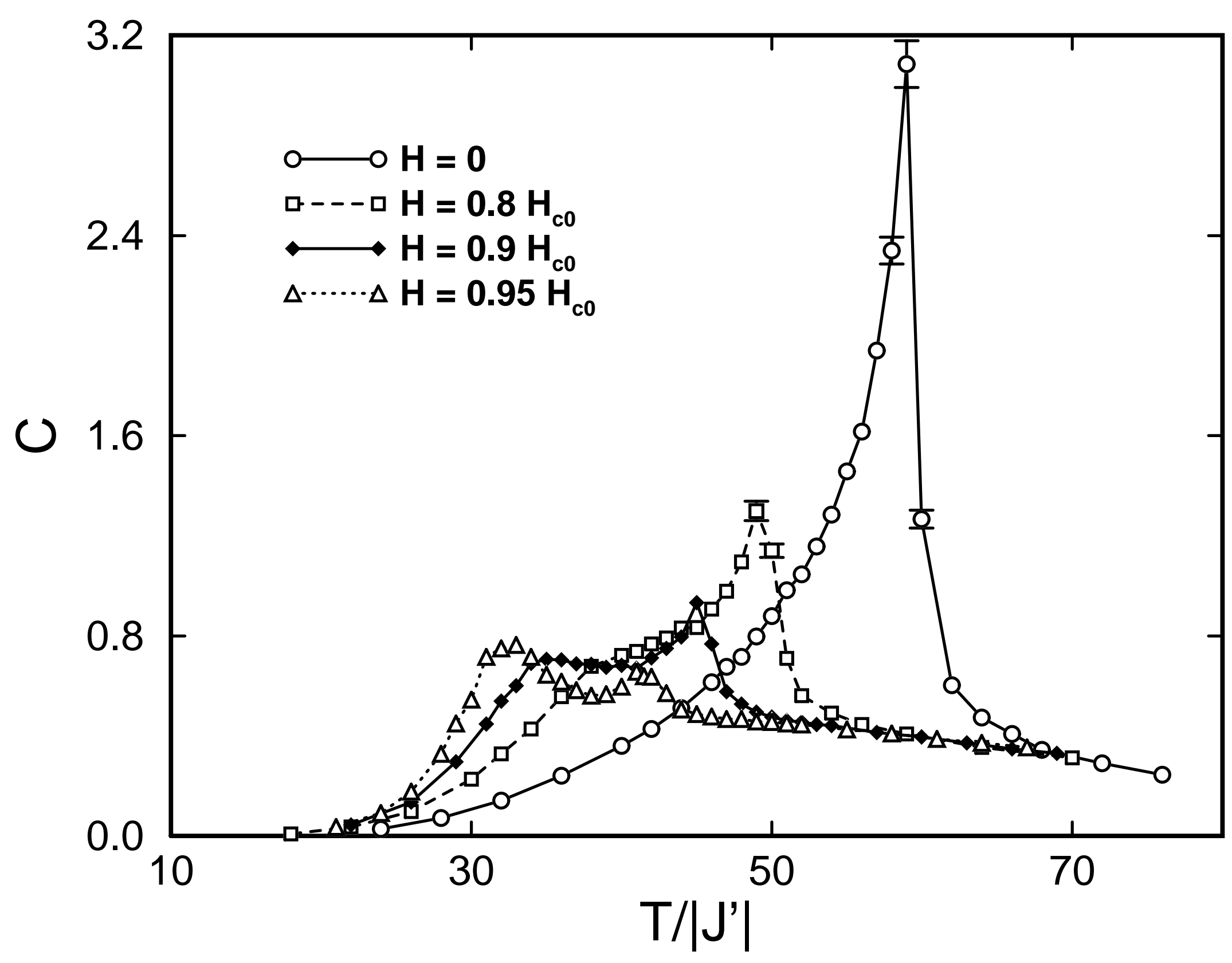


Pleimling/Selke: FIGURE 7

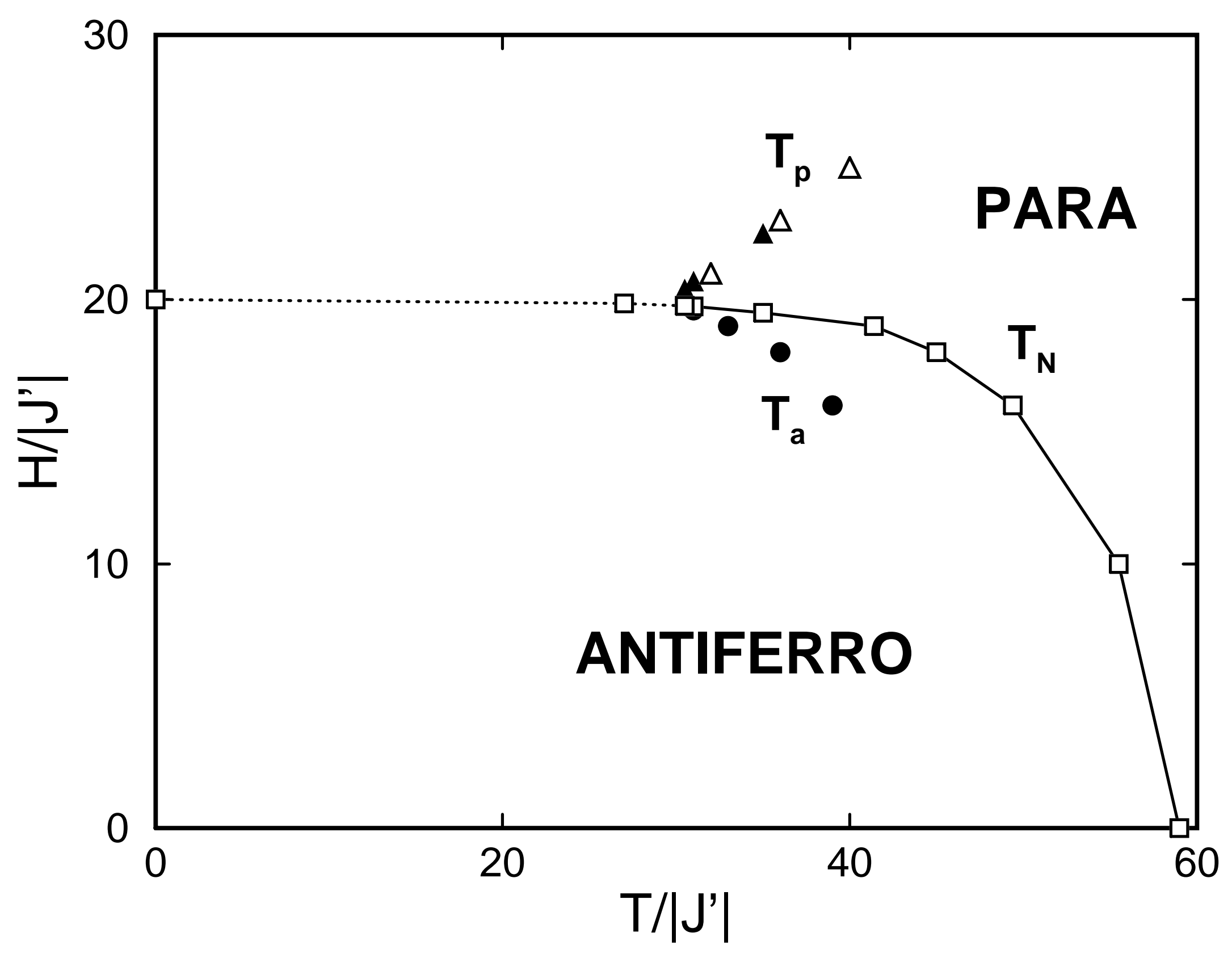




\section{Pleimling/Selke: FIGURE 8}

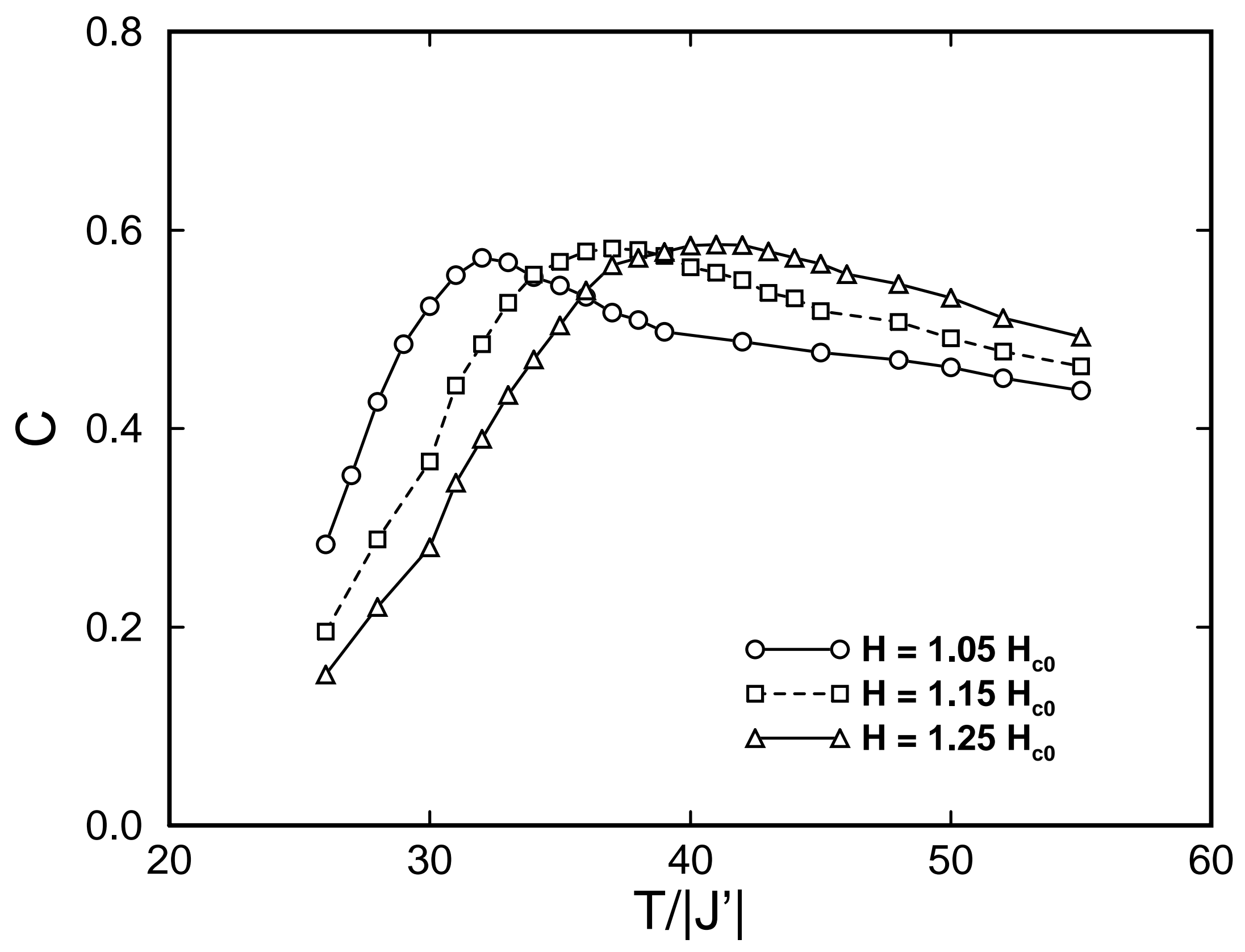




\section{Pleimling/Selke: FIGURE 9}

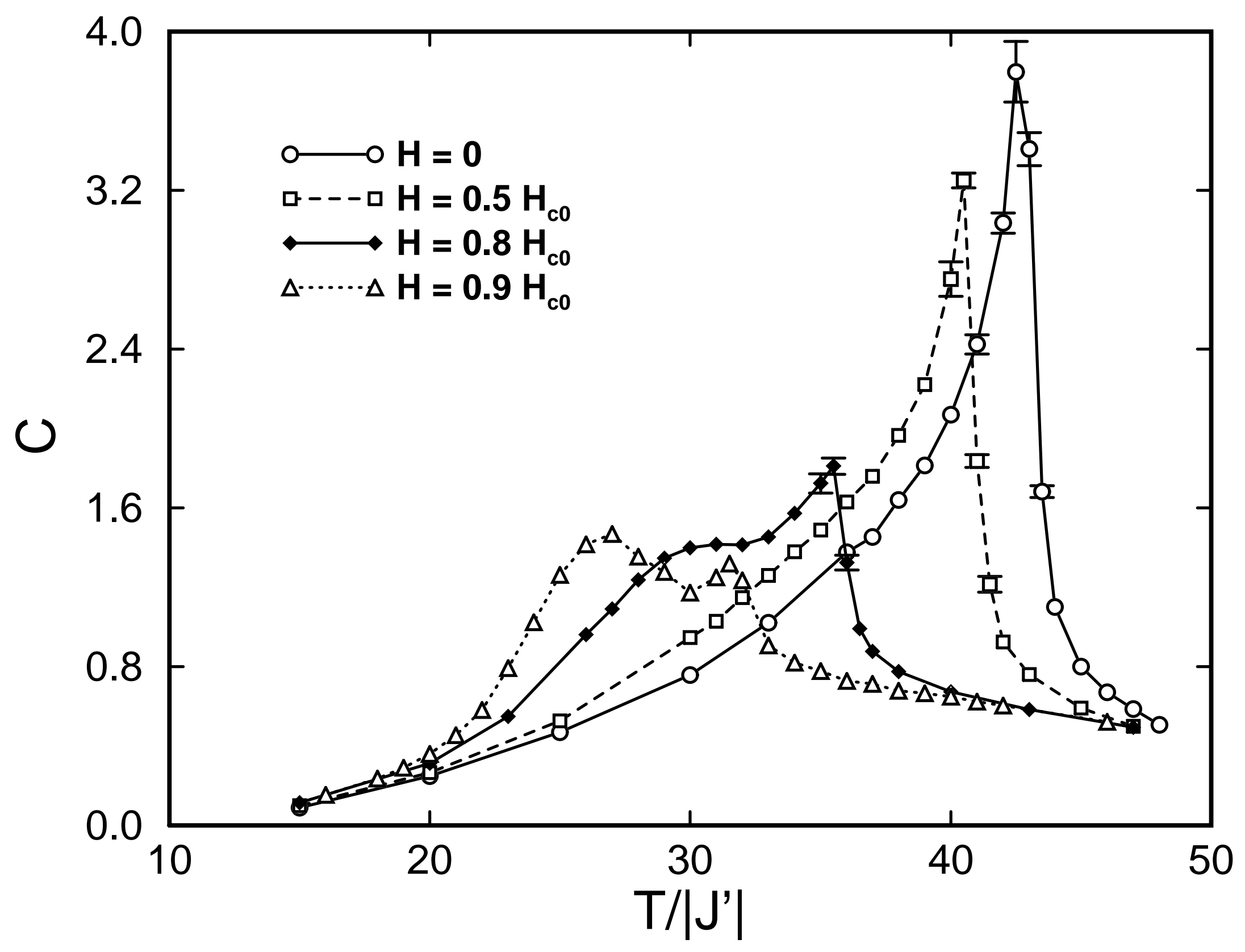




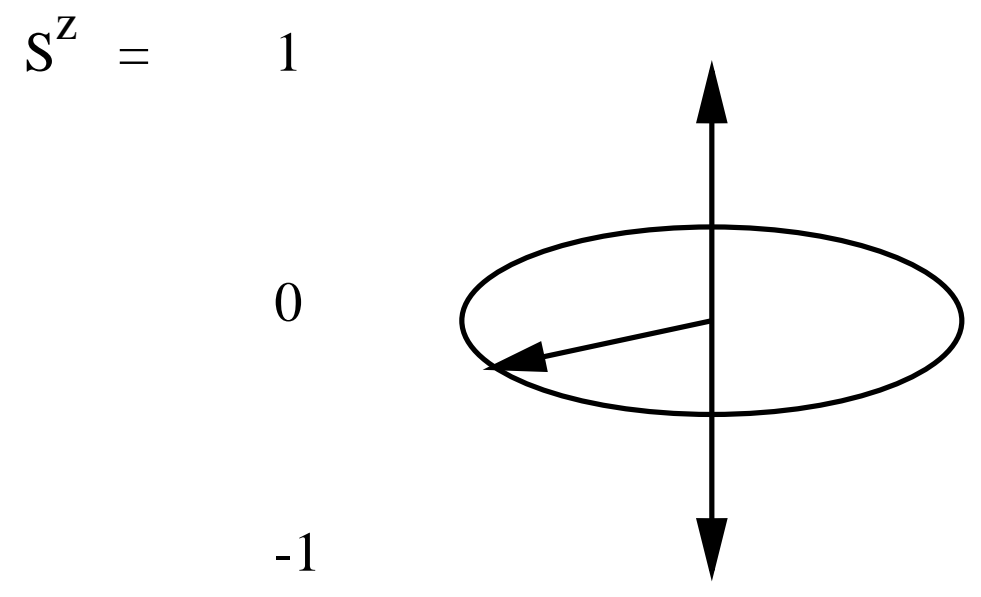

Pleimling/Selke: FIGURE 10 
Pleimling/Selke: FIGURE 11

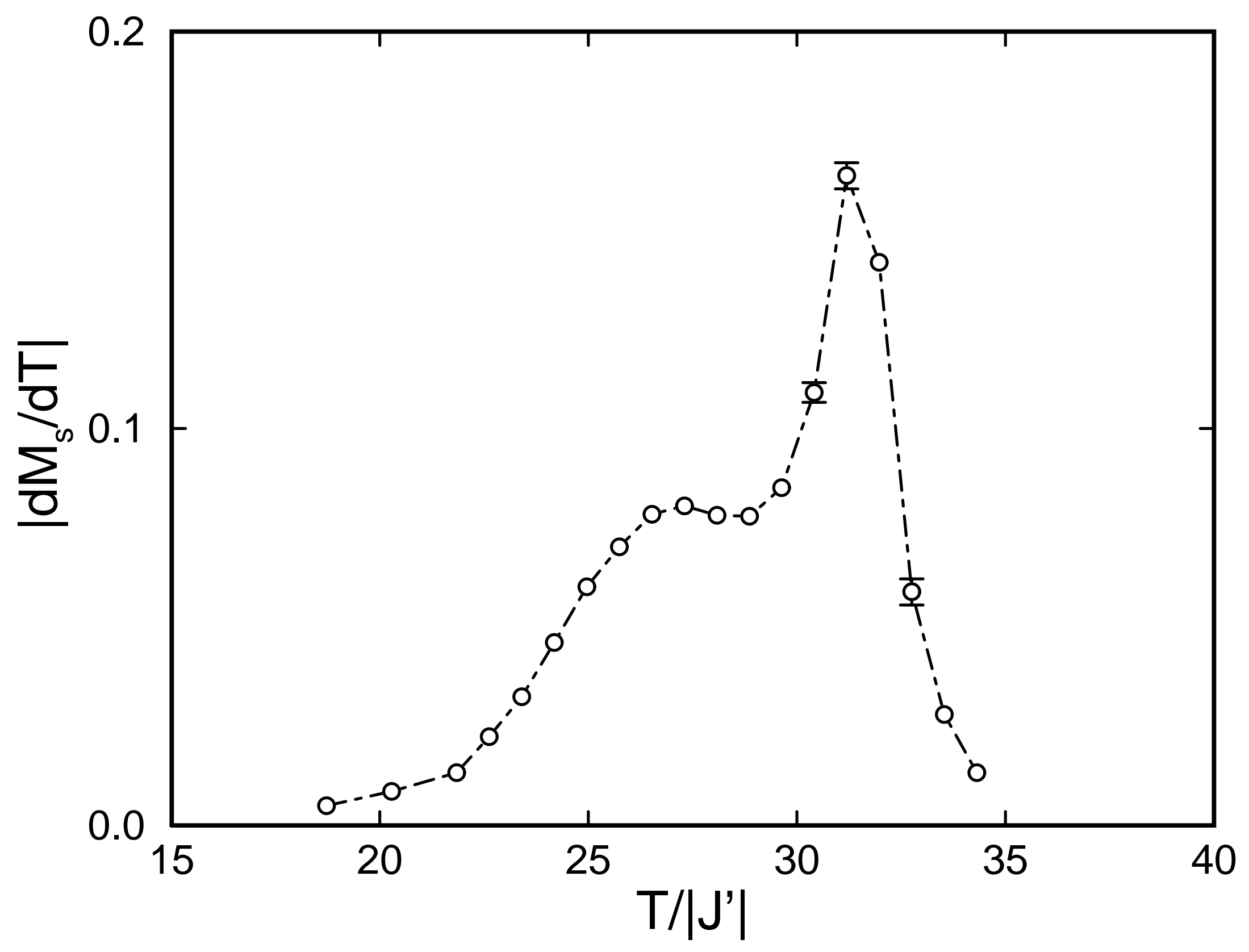


Pleimling/Selke: FIGURE 12a

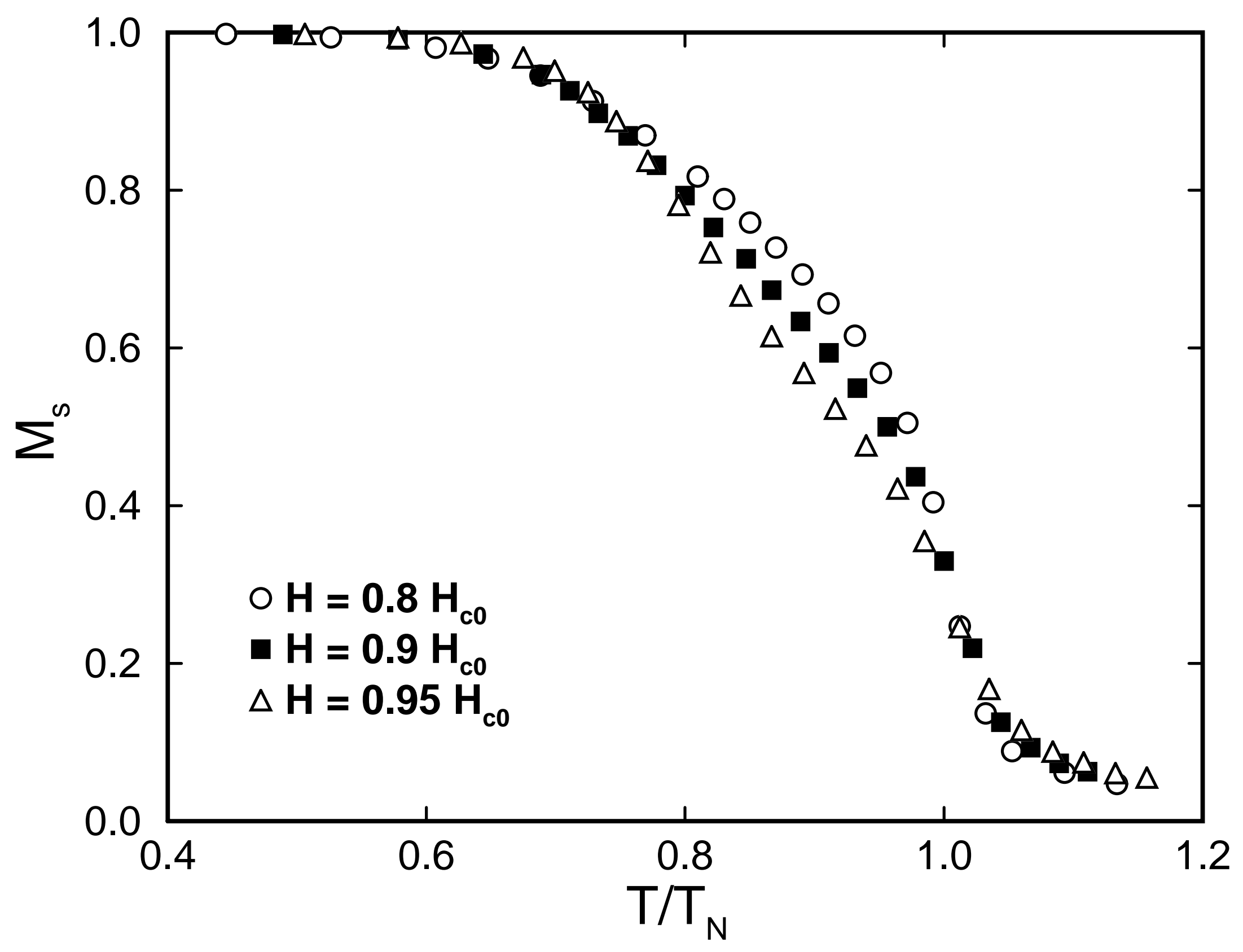


Pleimling/Selke: FIGURE 12b

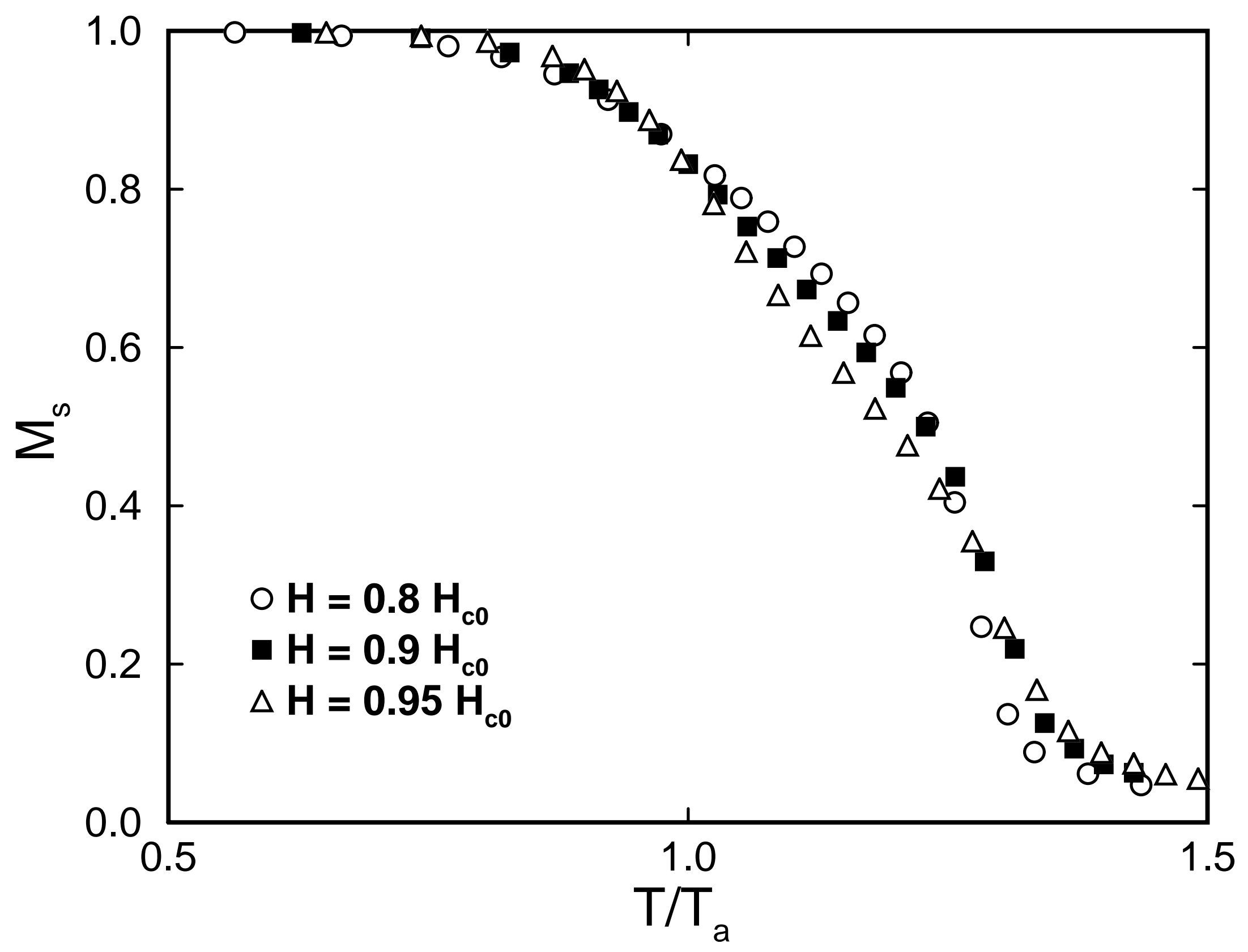

